\title{
Fuzzy Logic Based Decision System For PCB Defects Correction
}

\author{
Yogesh Kumar \\ Electronics \& Communication Engineering Department, Greater Noida Institute of Technology, Greater Noida, \\ INDIA
}

\begin{abstract}
Printed circuit boards (PCB) are vital and essential part for all of the electronics industries. It helps to minimize the size, ease of automation, increasing durability and reliability of any circuit being developed. Defect \& errors while developments are much obvious. Detecting them is primary part but taking decision for correction of same in testing specimen will be effective or not is much more crucial. Sometimes defect correction in the PCB is much more efficient then reprinting, in terms of time, resource and cost with respect to production. Making decision manually is tiresome \& less efficient. So in this paper a novel method of decision making system based on fuzzy logic is proposed which takes decision whether the testing specimen should undergo correction or reprinting. Fuzzy based system takes decision in the way humans do. Results shown for the proposed system are quite promising in decision making.
\end{abstract}

Keywords: defect correction, fatal errors, mamdani fuzzy, misalignment \& potential errors.

\section{INTRODUCTION}

The Printed Circuit Board (PCB) is very important in all electronic gadgets, which are used either for domestic use, or for industrial purpose. PCB design services are used to design the electronic circuits. Apart from electrically connecting, it also gives mechanical support to the electrical components. The PCB designs can be created both manually and automatically. Manual layouts are created with the help of CAD drafting, and the automatic router helps in the creation of the designs automatically. The designers usually prefer the manual way of designs, since they can implement their own ideas and techniques in them.

Non-contact methods can be from a wide range of selection from $\mathrm{x}$-ray imaging, ultrasonic imaging, thermal imaging and optical inspection using image processing [5-6]. Although these techniques are successful in detecting defects, none is able to classify the defects. In a non-contact reference based, image processing approaches template of a defect free PCB image and a defected test PCB image are segmented and compared with each other using image subtraction and other procedures. This project utilizes a non-contact reference based, image processing approach for defect detection and classification and simple image processing algorithm for locating those defects on PCB board. A template of a defect free PCB image and a defected test PCB image are segmented and compared with each other using image subtraction and other procedures. Discrepancies between the images are considered defects and are classified based on similarities and area of occurrences. After obtaining patterns concerning the results these are located on the PCB. This paper is organized as follows. Section 2 defines the review of previous works and research methodology chosen for this paper. Section 3 describes the details of mathematical and conceptual modeling of the proposed system for automatic decision of PCB defects. Section 4 contains the experimental results for defect detection and classification while the discussion and conclusion is described in section 5 .

\section{BACKGROUND}

Mogantiet al. [2] proposed three categories of inspection algorithm: referential approaches, nonreferential approaches, and hybrid approaches. The reference approaches is performed, by doing a comparison between the template $\mathrm{PCB}$ image and tested PCB image. There are two major techniques; image comparison techniques and model based inspection. Image comparison, which is the simplest approach, consists of comparing both images pixelby-pixel using simple logic operators such as XOR. The main difficulty found in these techniques is determining a precise alignment of the reference image and the test image, which makes its utilization difficult. More sophisticated proposals under the same idea, involve feature and template matching [2], but suffer from the same problem and normally require a large number of templates. This technique can be subdivided to image subtraction, template matching, and phase only method. Model-based methods are techniques, which match the pattern under inspection with a set of predefined models. They are also called Graph- Matching Methods [2] and are based on the structural, topological, and geometrical properties of the image. The major difficulty of those methods is related to the matching complexity. Although Sunet al.[4] proposed a 
technique called Pattern Attributed Hyper graph to make the method more practical, it still remains a complex and time consuming method. This technique can be subdivided to tree, syntactic, and graph matching method.

Human operators simply inspect visually against prescribed standards. The decisions made by them often involve subjective judgment, in addition to being labor intensive and therefore costly, whereas automatic inspection systems remove the subjective aspects and provide fast, quantitative dimensional assessments. Due to the following criteria, the sophistication in automated visual inspection has become a part of the modern manufacturing environment.

- They relieve human inspectors of the tedious jobs involved.

- Manual inspection is slow, costly, leads to excessive scrap rates, and does not assure high quality.

- Multi-layer boards are not suitable for human eyes to inspect.

- With the aid of a magnifying lens, the average fault- finding rate of a human being is about $90 \%$. However, on multi-layered boards (say 6 layered), the rate drops to about $50 \%$. Even with fault free power and ground layers, the rate does not exceed $70 \%$ [9].

- Industry has set quality levels so high that sampling inspection is not applicable.

- Production rates are so high that manual inspection is not feasible.

- Tolerances are so tight that manual visual inspection is inadequate.

A variety of approaches for automated optical inspection of printed circuit boards (PCBs) have been reported over the last two decades.

\section{PROPOSED WORK}

Making decision for correction of error in given specimen is quite significant because it can save resources as well as time. If the specimen cannot be corrected then whole printing process must be repeated for correct circuit board. Although manual decision for each defected PCB is tedious and less efficient; it can also cost resource and time wastage. A novel method for automatic decision system for such scenario is proposed in this paper. Proposed system is based on fuzzy logic which is efficient to take decisions the way humans do and quite fast. Block diagram of the system is shown in figure 1. For the correction decision first of all we need to compute the defects in the PCB. Reference image corresponds to the original circuit diagram, ideally which should be exactly printed on all of PCB's. Test image corresponds to the image of the board which is printed by the machine and should be compared or tested against the reference image. Before any kind of testing procedure the reference and test images must be noise free. This noise is in existence because of the various reasons but primary it is from the imaging device used to capture these images.

To remove the noise median filter (low pass filter) is used.

$\mathrm{y}[\mathrm{m}, \mathrm{n}]=\operatorname{median}\{\mathrm{x}[\mathrm{i}, \mathrm{j}],(\mathrm{i}, \mathrm{j}) \varepsilon \mathrm{w}\}$

equation (1)

where $\mathrm{w}$ represents a neighborhood defined by the user, centered around location $[\mathrm{m}, \mathrm{n}]$ in the image. As per equation (1) given below are the required pixels. Neighborhood pixels in increasing order are: $115,119,120,123, \mathbf{1 2 4}, 125,126,127$ and 150. Median value: 124. De-noised images are converted in to the binary images so that all of the pixels should have the values in form of 0 and 1 only, where 1 represents the white color and 0 represents the black color.

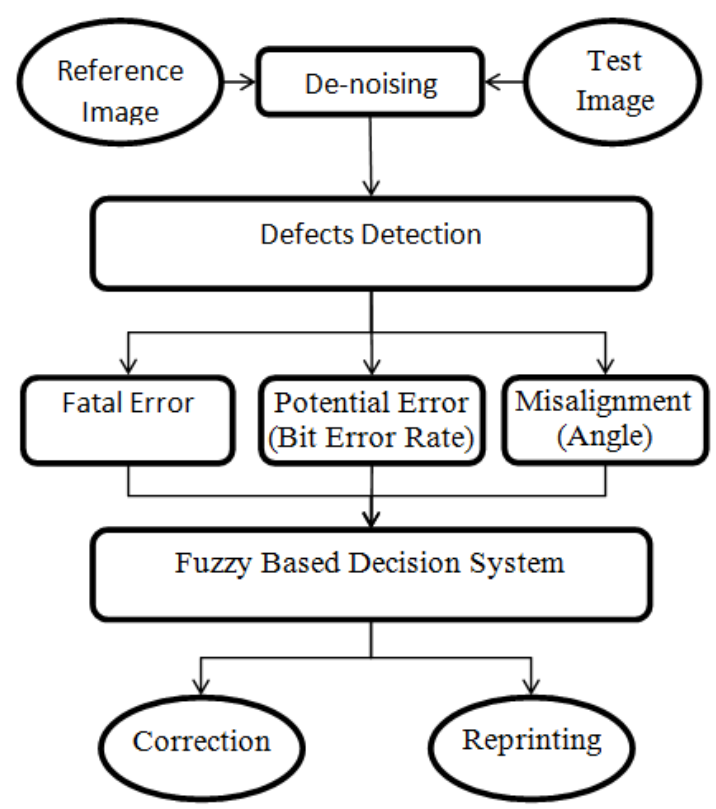

Fig. 1: Block diagram proposed method

Misalignment errors in the PCB test images are corrected using the SURF (Speed Up Robust Features) algorithm which is based on multi-scale space theory and the feature detector is based on Hessian matrix.

Since Hessian matrix has good performance and accuracy. In image $\mathrm{I}, \mathrm{x}=(\mathrm{x}, \mathrm{y})$ is the given point, the Hessian matrix $\mathrm{H}(\mathrm{x}, \sigma)$ in $\mathrm{x}$ at scale $\sigma$, it can be defined as:

$$
H(x, \sigma)=\left[\begin{array}{ll}
L_{x x}(x, \sigma) & L_{x y}(x, \sigma) \\
L_{y x}(x, \sigma) & L_{y y}(x, \sigma)
\end{array}\right] \quad \text { equation (2) }
$$

Where $\mathrm{L}_{\mathrm{xx}}(\mathrm{x}, \sigma)$ is the convolution result of the second order derivative of Gaussian filter $\frac{g^{2}}{d x^{2}} g(\sigma)$ with the image $\mathrm{I}$ in point $\mathrm{x}$, and similarly 
for $\mathrm{L}_{\mathrm{xy}}(\mathrm{x}, \sigma)$ and $\mathrm{L}_{\mathrm{yy}}(\mathrm{x}, \sigma)$. Surf features provides the angle and scaling factor for test image with respect to reference image to be corrected and are also shown in the results and discussion section.

Fatal \& potential errors are detected by XOR operations of test images with reference image. Subtraction of test image from reference image provides the potential and under printing errors, whereas the vice versa process provides the fatal and over printing errors.

Basically three values are obtained from the above methods of defect detection.

1) Bit error rate for fatal error (FBER)

2) Bit error rate for the potential error (PBER)

3) Misalignment angle (MisA)

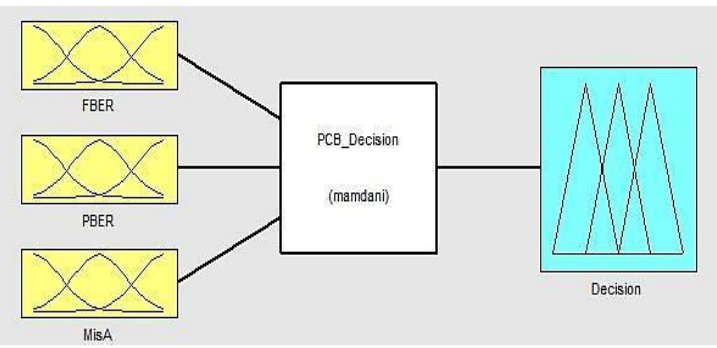

Fig. 2: Overview of proposed fuzzy system.

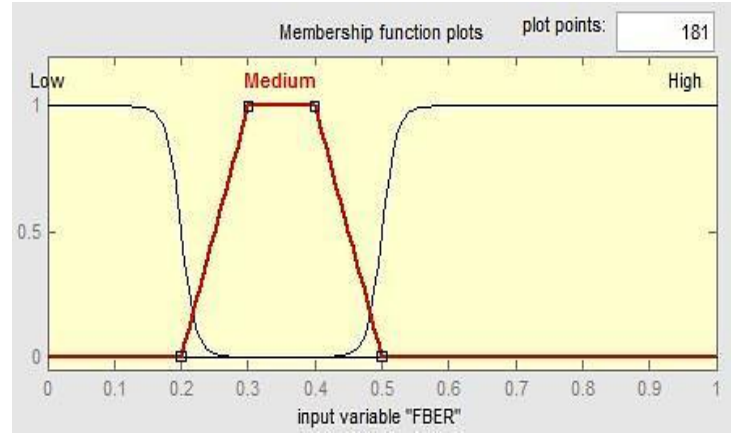

Fig. 3: Membership function of FBER

On the basis of these three inputs proposed system takes the decision that the specimen should undergo correction or reprinting. Output of the proposed system is in the range of 0 to 1 . So a threshold is set as 0.5 below which the specimen should undergo correction and above which it means that specimen must go for reprinting. Figure 2 represents the overview proposed fuzzy system.

While designing the system and method is min, or method is max, Implication is min, Aggregation is max and de-fuzzification method is taken as centroid. In the figure 2, FBER represents the bit error rate of the fatal errors; PBER represents the bit error rate for the potential errors; MisA represents the angle for misalignment and Decision represents the output. Membership functions for above three inputs and one output are shown in figure 3 , figure 4 , figure 5 and figure 6 respectively.
Misalignment angle can be in range of 0 to $360^{\circ}$ but the membership function range is given from 0 to 27 . Because the algorithm used for misalignment error correction can correct the errors in range of $45^{\circ}$ steps from $0^{\circ}$ to $360^{\circ}\left(45^{\circ}, 90^{\circ}, 135^{\circ}\right.$, $180^{\circ}, 225^{\circ}, 270^{\circ}, 315^{\circ}$ and $360^{\circ}$ ) and scaling factor of 0 to 2 . So the value taken for misalignment angle input is the difference from nearest angle from the list provided above.

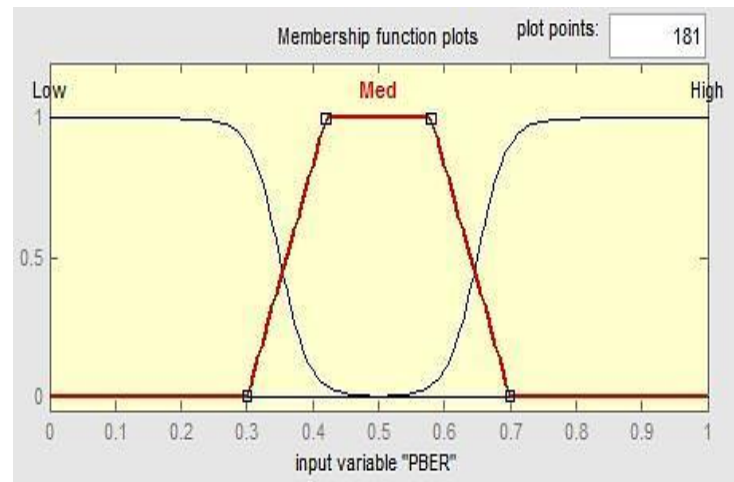

Fig. 4: Membership function of PBER

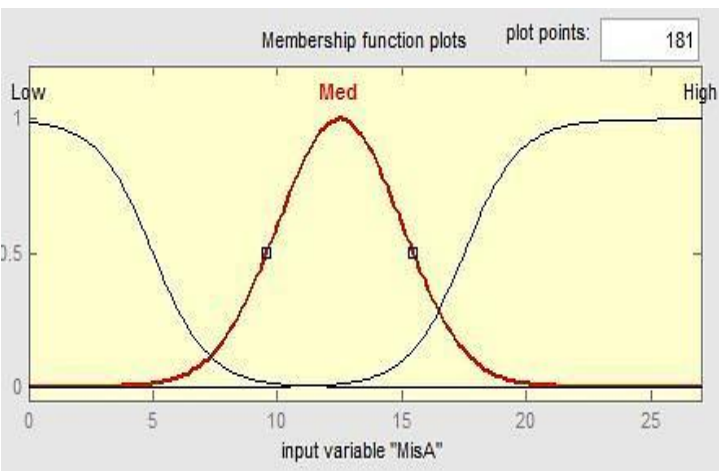

Fig. 5: Membership function of MisA

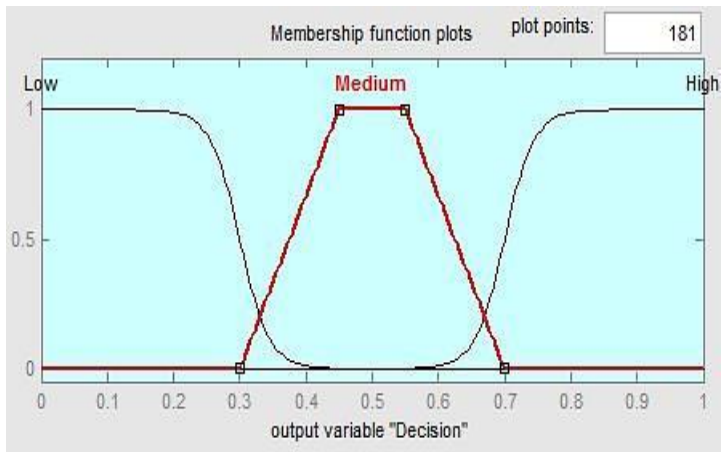

Fig. 6: Membership function of Decision

For the three input system, each input having 3 membership functions; as per combination there are 27 rules for the system which are given below:

1. If (FBER is High) and (PBER is Low) and (MisA is Med) then (Decision is High) (1)

2. If (FBER is High) and (PBER is Low) and (MisA is Low) then (Decision is High) (1) 
3. If (FBER is High) and (PBER is Low) and (MisA is High) then (Decision is High) (1)

4. If (FBER is High) and (PBER is High) and (MisA is Med) then (Decision is High) (1)

5. If (FBER is High) and (PBER is High) and (MisA is Low) then (Decision is High) (1)

6. If (FBER is High) and (PBER is High) and (MisA is High) then (Decision is High) (1)

7. If (FBER is High) and (PBER is Med) and (MisA is Med) then (Decision is High) (1)

8. If (FBER is High) and (PBER is Med) and (MisA is Low) then (Decision is High) (1)

9. If (FBER is High) and (PBER is Med) and (MisA is High) then (Decision is High) (1)

10. If (FBER is Low) and (PBER is Low) and (MisA is Low) then (Decision is Low) (1)

11. If (FBER is Low) and (PBER is Low) and (MisA is Med) then (Decision is Medium) (1)

12. If (FBER is Low) and (PBER is Low) and (MisA is High) then (Decision is High) (1)

13. If (FBER is Low) and (PBER is Med) and (MisA is Low) then (Decision is Low) (1)

14. If (FBER is Low) and (PBER is Med) and (MisA is Med) then (Decision is Medium) (1)

15. If (FBER is Low) and (PBER is Med) and (MisA is High) then (Decision is High) (1)

16. If (FBER is Low) and (PBER is High) and (MisA is Low) then (Decision is Medium) (1)

17. If (FBER is Low) and (PBER is High) and (MisA is Med) then (Decision is High) (1)

18. If (FBER is Low) and (PBER is High) and (MisA is High) then (Decision is High) (1)

19. If (FBER is Medium) and (PBER is Low) and (MisA is Low) then (Decision is Medium) (1)

20. If (FBER is Medium) and (PBER is Low) and (MisA is Med) then (Decision is High) (1)

21. If (FBER is Medium) and (PBER is Low) and (MisA is High) then (Decision is High) (1)

22. If (FBER is Medium) and (PBER is Med) and (MisA is Low) then (Decision is Medium) (1)

23. If (FBER is Medium) and (PBER is Med) and (MisA is Med) then (Decision is High) (1)

24. If (FBER is Medium) and (PBER is Med) and (MisA is High) then (Decision is High) (1)

25. If (FBER is Medium) and (PBER is High) and (MisA is Low) then (Decision is High) (1)

26. If (FBER is Medium) and (PBER is High) and (MisA is Med) then (Decision is High) (1)

27. If (FBER is Medium) and (PBER is High) and (MisA is High) then (Decision is High) (1)

These rules map each and every possibility of the inputs as per their combination. Figure 7 represents the surface view plot for PBER, FBER and Decision of the system. Figure 8 represents the relationship plot between MisA and Decision. Variation of input and out parameters can be analytically analyzed using these plots.

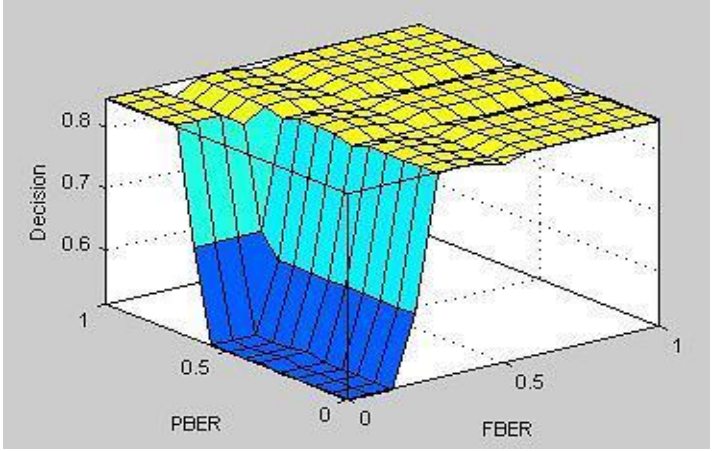

Fig. 7: Relationship between PBER, FBER and Decision.

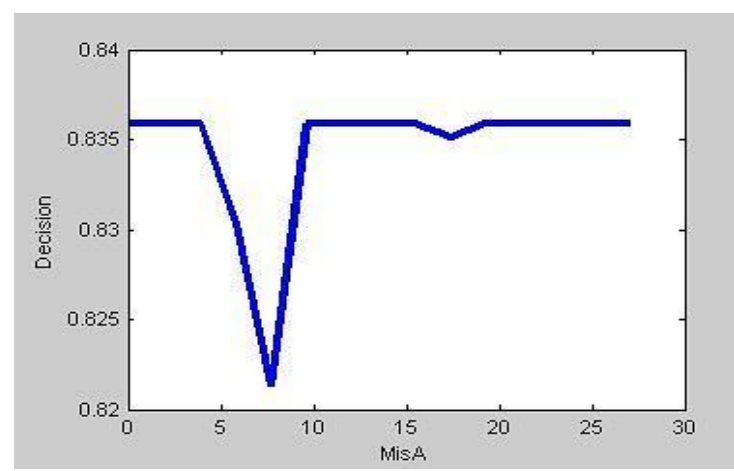

Fig. 8: Relationship between MisA and Decision.

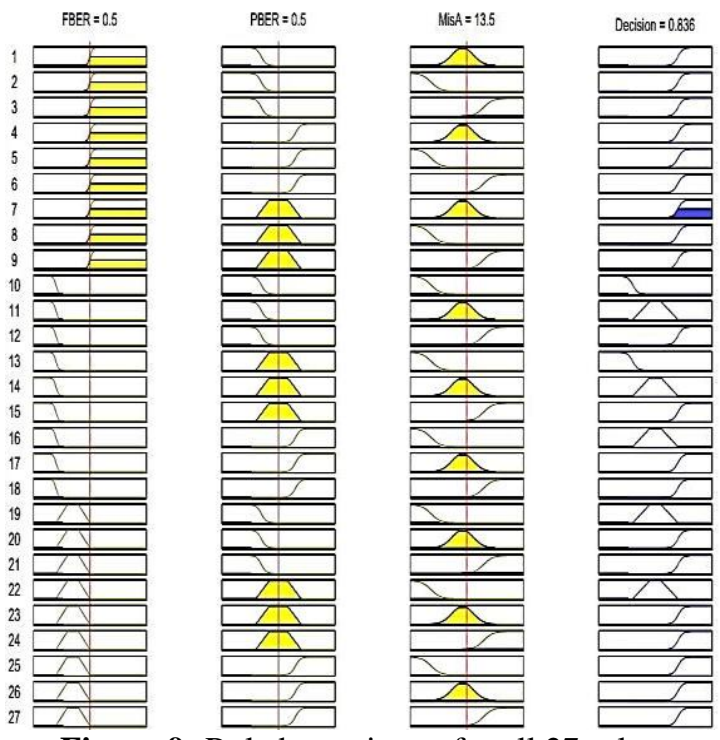

Figure 9: Rule base viewer for all 27 rules.

\section{RESULTS \& DISCUSSION}

Given below are the test images and results by the proposed algorithm. For the test image-I results along with reference images are shown below. Figure 10 represents the reference image, figure 11 represents the test image and figure 12 represents fatal and potential errors. Exact location of the errors are also provided in the excel file for exact localization. Table-I represents the results for 20 samples. 


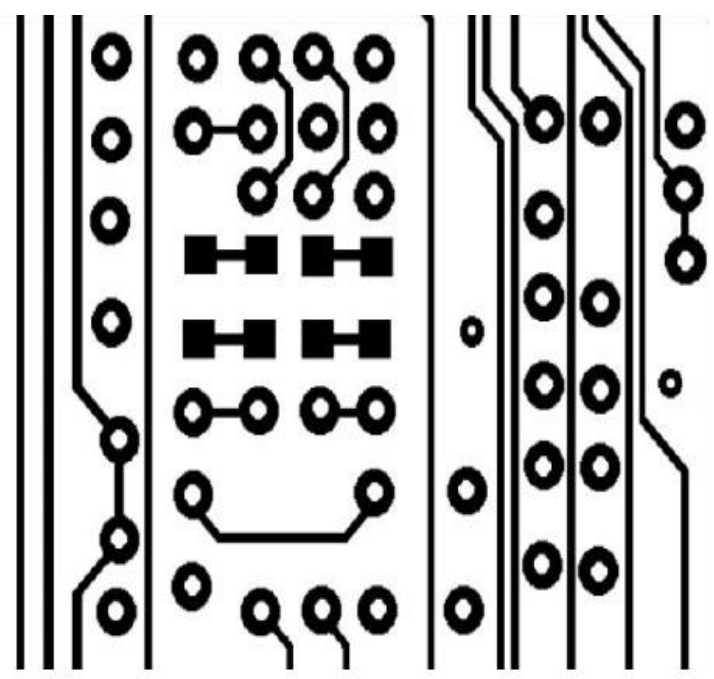

Figure 10: Reference Image-1.

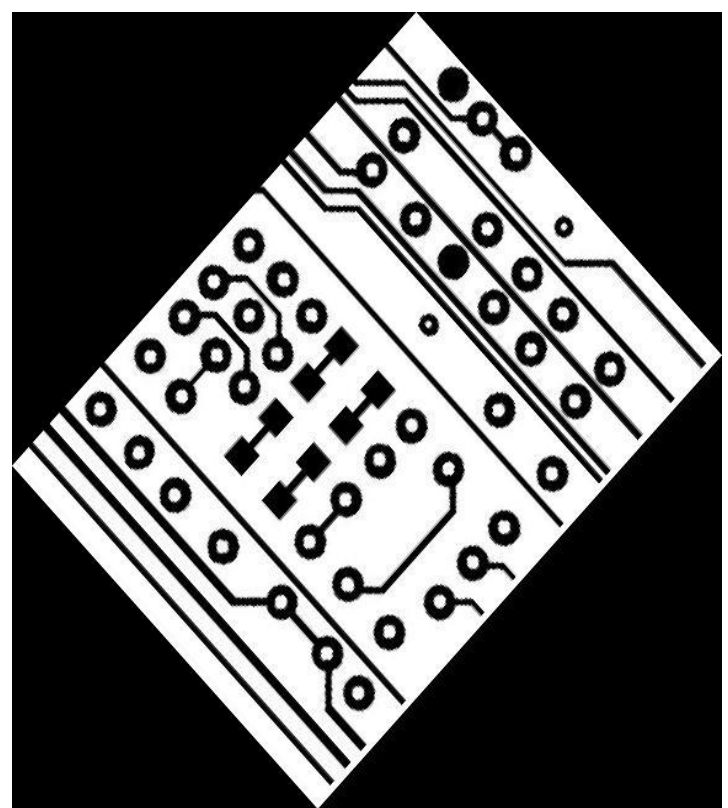

Figure 11: Test Image-1.

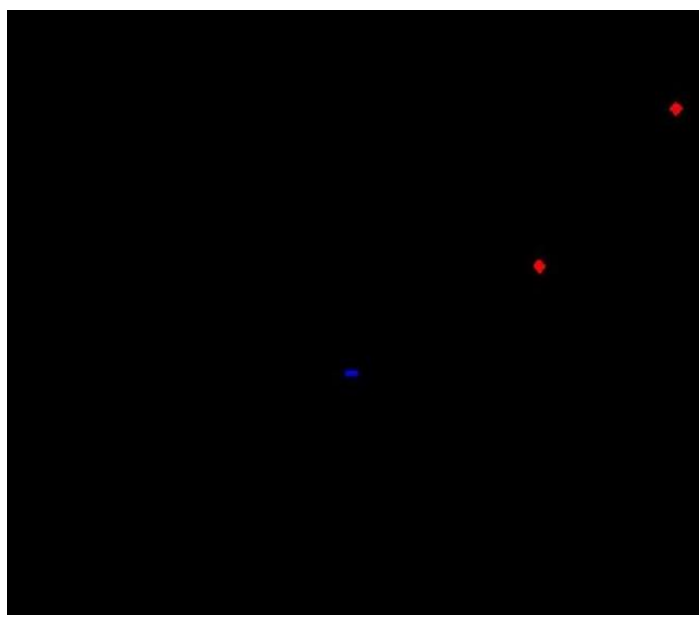

Figure 12: Fatal (Red) \& Potential (Blue) Error in test image-1
TABLE I. Results

\begin{tabular}{|l|l|l|l|l|l|l|}
\hline Sr. & F BER I/P-1 & P BER I/P-2 & Angle & Diff. I/P-3 & Fuzzy Result & Decision \\
\hline 1 & 0.075 & 0.64 & 274 & 9 & 0.65 & Reprint \\
\hline $\mathbf{2}$ & 0.055 & 0.044 & 224 & 4 & 0.16 & Correct \\
\hline $\mathbf{3}$ & 0.013 & 0.091 & 300 & 10 & 0.494 & Correction \\
\hline $\mathbf{4}$ & 0.024 & 0.057 & 291 & 19 & 0.829 & Reprint \\
\hline $\mathbf{5}$ & 0.41 & 0.85 & 133 & 3 & 0.846 & Reprint \\
\hline 6 & 0.031 & 0.075 & 90 & 5 & 0.171 & Correct \\
\hline 7 & 0.022 & 0.13 & 66 & 19 & 0.829 & Reprint \\
\hline 8 & 0.046 & 0.093 & 168 & 7 & 0.304 & Correct \\
\hline 9 & 0.084 & 0.079 & 330 & 20 & 0.843 & Reprint \\
\hline 10 & 0.6 & 0.69 & 275 & 10 & 0.839 & Reprint \\
\hline 11 & 0.076 & 0.55 & 231 & 11 & 0.503 & Reprint \\
\hline 12 & 0.002 & 0.026 & 60 & 20 & 0.843 & Reprint \\
\hline 13 & 0.047 & 0.084 & 291 & 19 & 0.829 & Reprint \\
\hline 14 & 0.09 & 0.61 & 327 & 17 & 0.717 & Reprint \\
\hline 15 & 0.1 & 0.004 & 279 & 14 & 0.518 & Reprint \\
\hline 16 & 0.075 & 0.096 & 2 & 3 & 0.155 & Correct \\
\hline 17 & 0.029 & 0.56 & 23 & 17 & 0.717 & Reprint \\
\hline 18 & 0.016 & 0.066 & 328 & 18 & 0.793 & Reprint \\
\hline 19 & 0.065 & 0.058 & 65 & 20 & 0.843 & Reprint \\
\hline 20 & 0.065 & 0.18 & 233 & 13 & 0.507 & Reprint \\
\hline
\end{tabular}

\section{CONCLUSION}

Fatal, Potential and Misalignment errors can be detected easily using surf features for any value of angle. These three inputs are fed to the proposed fuzzy system which evaluates the decision on the bases of rule base provide. By the output of the system one can easily decide that given specimen should undergo Reprinting or correction. Proposed system provides fast, efficient and promising results.

Further area of improvisation can be automatic correction of errors for the specimens which are needed to undergo correction.

\section{REFERENCES}

[1]. J. Hong, K. Park, and K. Kim, "Parallel Processing Machine Vision System for Bare PCB Inspection", in Proceedings of the 24th Annual Conference of the IEEE Industrial Electronics Society, 1998, 3, 1346-1350.

[2]. M. Moganti, F. Ercal, C. H. Dagli, and T. Shou, "Automatic PCB Inspection Algorithms: A Survey", Computer Vision and Image Understanding, 63 (2), 1996, 287-313.

[3]. R. S. Guh and J. D. T. Tannock, "A Neutral Network Approach to Characterize Pattern Parameters in Process Control Charts" ,Journal of Intelligent Manufacturing,10(5), 1996, 449-462.

[4]. Y. Sun and C. Tsai, "A New Model-Based Approach for Industrial Visual Inspection", Pattern Recognition, 25, 1992, 1327-1336.

[5]. W. Wu, M. J. Wang and C. M. Liu, "Automated Inspection of Print Circuit Boards through Machine Vision", Computers in Industry, 28, 1996, 103-111. 
[6]. R. Heriansyah and S. A. R. Abu-Bakar, "Defects Classification on Bare PCB using Multiple Learning Vector Quantization Neural Network Paradigm", in International Conference on Computer Graphics, Imaging, and Visualization , 2004, 50-53.

[7]. N. K. Khalid and Z. Ibrahim, "An Image Processing Approach Towards Classification of Defects on Printed Circuit Board", in Malaysia-Japan International Symposium on Advanced Technology, 2007.

[8]. N. K. Khalid, Z. Ibrahim, and M. S. Zainal Abidin, "An Algorithm to Group Defects on Printed Circuit Board for Automated Visual Inspection", International Journal of Simulation: Systems, Science, and Technology, 9(2), 2008, 1-10.

[9]. P. C. Chang, L. Y. Chen, and C. Y. Fan, “A Case-Based Evolutionary Model for Defect Classification of Printed Circuit Boards Images", Journal of Intelligent Manufacturing, 19, 2008, 203-214.

[10]. M. H. Tatibana and R. de A. Lotufo, "Novel Automatic PCB Inspection Technique Based on Connectivity", Computer Graphics and Image Processing Proceedings, 1997, 187-194

[11]. Suhasini A ,Sonal D Kalro , Prathiksha B G, Meghashree B S , Phaneendra H D, "PCB Defect Detection Using Image Subtraction Algorithm", International Journal of Computer Science Trends and Technology (IJCST) - Volume 3 Issue 3, ISSN: 2347-8578 www.ijcstjournal.org May-June 2015.

[12]. "Printed circuit board defect detection using mathematical morphology and MATLAB image processing tools", Researchgate Article, DOI: 10.1109/ICETC.2010.5530052, January 2010.

[13]. Takumi Uemura, Gou Koutaki and Keiichi Uchimura, "Image Segmentation based on Edge Detection using Boundry Code", International journal of innovative computing, information and computing, Volume 7, Number 10, October 2011.

[14]. E. Argyle. "Techniques for edge detection," Proc. IEEE, vol. 59, pp. 285-286, 2012.

[15]. F. Bergholm. "Edge focusing," in Proc. 8th Int. Conf. Pattern Recognition, Paris, France, pp. 597- 600, 2013. 